\title{
Statistical Asymmetry-based Brain Tumor Segmentation from 3D MR Images
}

\author{
Chen-Ping $\mathrm{Yu}^{1}$, Guilherme C. S. Ruppert ${ }^{23}$, Dan T. D. Nguyen ${ }^{4}$, \\ Alexandre X. Falcao ${ }^{2}$, and Yanxi Liu ${ }^{1}$ \\ 1 Department of Computer Science and Engineering, Pennsylvania State University, \\ 342 Information Sc. and Tech. Building, University Park, PA, USA 16802 \\ cpu1982@gmail.com, yanxi@cse.psu.edu \\ 2 Institute of Computing, University of Campinas, Av. Albert Einstein 1251, \\ Campinas, Brazil 13083-852 \\ \{ruppert, afalcao\}@ic.unicamp.br \\ ${ }^{3}$ Renato Archer Center for Information Technology, Rod. Dom Pedro km143, \\ Campinas, Brazil 13069-901 \\ guilherme.ruppertecti.gov.br \\ ${ }^{4}$ Department of Radiology, Penn State Hershey Medical Center, 500 University Drive, \\ Hershey, PA, USA 17033 \\ dnguyen1@hmc.psu.edu
}

\begin{abstract}
The precise segmentation of brain tumors from MR images is necessary for surgical planning. However, it is a tedious task for the medical professionals to process manually. The performance of supervised machine learning techniques for automatic tumor segmentation is time consuming and very dependent on the type of the training samples. Brain tumors are statistically asymmetrical blobs with respect to the mid-sagittal plane (MSP) in the brain and we present an asymmetry-based, novel, fast, fully-automatic and unsupervised framework for 3D brain tumor segmentation from MR images. Our approach detects asymmetrical intensity deviation of brain tissues in 4 stages: (1) automatic MSP extraction, (2) asymmetrical slice extraction for an estimated tumor location, (3) region of interest localization, and (4) 3D tumor volume delineation using a watershed method. The method has been validated on 17 clinical MR volumes with a $71.23 \% \pm 27.68 \%$ mean Jaccard Coefficient.
\end{abstract}

\section{Introduction}

Brain tumors vary in size, shape, color, and location, which is precisely the reason why automatic tumor segmentation is challenging. While the medical professionals are able to hand label the optimal details of each tumor case, such task is incredibly tedious and time-consuming. Therefore, the need for tumors to be automatically segmented remains an unsolved problem in clinical practice.

In recent years, related unsupervised approaches that utilizes brain asymmetry [22] [9] requires human interaction to manually select a $2 \mathrm{D}$ slice of interest, and the $2 \mathrm{D} / 3 \mathrm{D}$ tumor segmentation from such analysis has not been shown to work fully automatically.

Markov Random Fields [15] and Conditional Random Field [12] based machine learning techniques have been applied in tumor segmentation tasks as well. Methods like Discriminative Random Fields [11], Support Vector Random Fields [13], and 

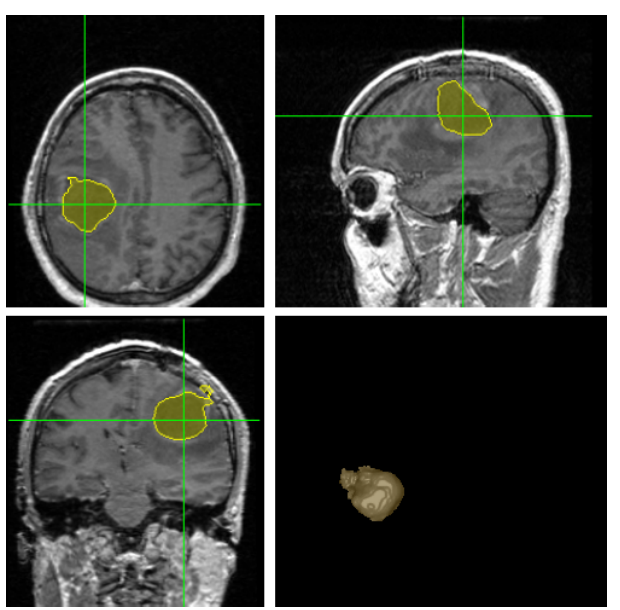

Fig. 1. Sample result for case 6 , showing the 3 orientations and the $3 \mathrm{D}$ view on the lower right portion of the figure, with Jaccard Coefficient of $90.11 \%$.

Pseudo-Conditional Random Field [14] have been shown to offer better performance. Other supervised statistical machine learning approaches include using fractal features [8], alignment features [23], one-class support vector machine [26], using Bayesian classifier [5], tumor localization using diagonal nearest-neighbors [7], segmentation by outliers [21], and high-dimensional features with level-set [3]. A recent supervised method proposed [27], though demonstrating promising results on small tumor detection using brain asymmetry, is not addressing 3D tumor segmentation problem.

In order to extract features to be used for pixel/voxel classification, standard machine learning methods must first register the input volume. The registration process usually takes hours of time while being a research area of its own [10]; the performance accuracy of the classification and segmentation result depends largely on the training samples and the pre-defined feature sets.

Unsupervised algorithms using the bilateral symmetry of the brain start to emerge in recent years [16][22]. However, unsupervised methods based on symmetry are still in its early stages as such methods are not yet fully automatic, and the accuracy also has a lot of room for improvements [16][22].

In this paper, we present a novel, fully automatic and unsupervised framework that is based upon an intuitive yet statistically justified observation that tumors are one of the most prominent asymmetrical blobs in the brain. We show that our approach is invariant to different types of tumor with the asymmetrical blob assumption, and we are able to automatically localize and delineate the tumors. The entire process is fast due to the unsupervised nature, taking about 3 minutes to run.

\section{Statistical Asymmetry-based Method}

Human brains are commonly accepted as statistically symmetrical with respect to its Mid-Sagittal Plane (MSP)[24]. Our proposed method takes the advantage of this prop- 


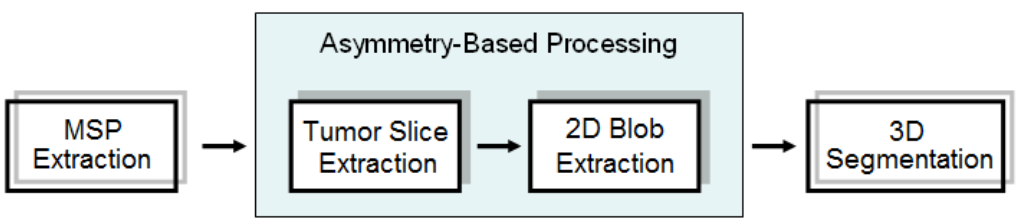

Fig. 2. Diagram of the entire process of the proposed 4-stage algorithm.

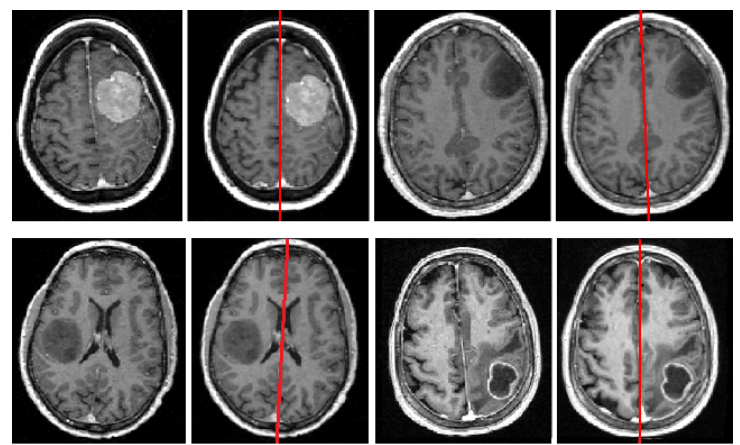

Fig. 3. Examples of results from Stage 1 (MSP extraction). The Figure shows 4 pairs (original image and the results from the method) for cases 1, 3, 4 and 10 with slice 184, 160, 161, and 156 respectively.

erty by processing the brain through asymmetry comparisons of its structural and pixel intensity distribution. We propose a 4-stage process: 1) MSP alignment, 2) locate an axial slice that contains parts of the tumor, 3 ) localize the 2D shape of the tumor from the extracted slice-of-interest (SOI), 4) grow the 3D shape of the tumor out bi-directionally.

\subsection{Stage 1: Automatic Mid-Saggital Plane Extraction}

Since this work is a symmetry-based method, it depends on the bi-lateral symmetry analysis of the brain, which requires the localization of the mid-sagittal plane (MSP) which is the reference of symmetry of the brain. Based on the MSP location, the image can be realigned, i.e. rotated and translated in a way so the MSP is found in the central slice of the image. In order to perform the MSP extraction, first, all input images were re-interpolated to isotropic voxels to restore the original proportion of the brain.

The method described in [24] was used to automatically locate the mid-sagittal plane (MSP). It is a very fast and accurate method for MSP extraction and is based on bi-lateral symmetry maximization. It uses cross-correlation of edges in the full 3D domain as the symmetry criteria and finds the plane that maximizes this criteria using an optimized multi-scale search algorithm. On average, the method takes less than $25 \mathrm{~s}$ to run on a modern desktop machine over a typical MRI data. Figure 3 shows some examples of the results from this method. 


\subsection{Stage 2: Slice of Interest (SOI) Extraction}

We formulate the problem using a Bayesian model,

$$
P\left(Z \mid S_{Z}\right) \propto P\left(S_{Z} \mid Z\right) \times P(Z)
$$

Our goal is to extract a 2D slice $E[S]$ from the volume of interest (VOI) that contains part of the tumor, where we obtain $E[S]$ from the posterior $P\left(Z \mid S_{Z}\right)$ by maximizing the conditional likelihood $P\left(S_{Z} \mid Z\right)$. We define $S_{Z}$ as the full count of axial-view slices from the neck towards the top of the head, where $S_{Z}=\left\{S_{L \mid Z}, S_{R \mid Z} \mid Z=\right.$ $\left.1, \ldots, z, L=l_{1}, \ldots, l_{u}, R=r_{1}, \ldots, r_{u}\right\}$, and a prior $P(Z)$ that models the likelihood of tumor location.

The brain is split into 2 halves along MSP of $S_{Z}$, where $S_{L \mid Z}$ and $S_{R \mid Z}$ are the left and right half. $S_{L \mid Z}$ and $S_{R \mid Z}$ are then further equally partitioned perpendicular to the MSP into $u$ pieces for the consideration of spatial information.

We compute an asymmetry score using Earth-Mover Distance [17] for each pair of $S_{L \mid Z}$ and $S_{R \mid Z}$ that is equally partitioned into $u$ pieces. The Earth-Mover Distance between two normalized histograms $H(A)$ and $H(B)$ is denoted here as $\Phi(H(A), H(B))$. The normalized 3-dimensional histogram (x,y location and the intensity level at each pixel) of each partitioned piece are denoted as $H_{3}\left(S_{l_{u} \mid Z}\right)$ and $H_{3}\left(S_{r_{u} \mid Z}\right)$. To determine how asymmetrically distributed are the intensity values of $S_{z}$ with its piece-wise spatial information, each pair of partitions' EMD asymmetry scores are summed to form the likelihood probability:

$$
P\left(S_{Z}\right)=\sum_{i=1}^{u} \Phi\left(H_{3}\left(S_{l_{u} \mid Z}\right), H_{3}\left(S_{r_{u} \mid Z}\right)\right)
$$

We obtain the likelihood probability from each slice $S_{z}$, which we can plot and treat as a 1D signal (Figure 4). We wish to locate the most asymmetrical slice. However, this signal can be noisy due to different parts of the brain (especially the neck region and the scalp top) and intensity variance, therefore we must apply a prior $P(Z)$ that models the likelihood of tumor location. We found that parts of the Inverse-Gamma distribution resembles the prior likelihood of tumor location well (Figure 4). We denote InverseGamma as $f(Z ; \alpha, \beta)$, and it is defined as:

$$
P(Z)=f(Z ; \alpha, \beta)=\frac{\beta^{\alpha}}{\Gamma(\alpha)}(1 / Z)^{\alpha+1} e^{-\beta / Z}
$$

The Inverse-Gamma prior formulates the likelihood $P\left(S_{Z}\right)$ into a conditional probability $P\left(S_{Z} \mid Z\right)$. Now we can calculate Bayesian posterior probability $P\left(Z \mid S_{Z}\right)$ from the originally obtained conditional likelihood signal $P\left(S_{Z} \mid Z\right)$ with the Inverse-Gamma prior $P(Z)$.

After $P\left(Z \mid S_{Z}\right)$ is computed, any posterior probability that is outside of $3 \sigma$ is reduced to the mean of the entire posterior probability and its $S_{Z-2}, S_{Z-1}, S_{Z+1}$, and $S_{Z+2}$ neighbors as a measure to remove outliers. The processed posterior probability $P\left(Z \mid S_{Z}\right)$ is convoluted by $1 \mathrm{D}$ Gaussian filter $N\left(\mu=0, \sigma^{2}=3\right)$ with horizontal size of 9 (spans 4 slices before and after the current position) for all observations to be weighted by their neighboring information as well as filtering out the high frequency 


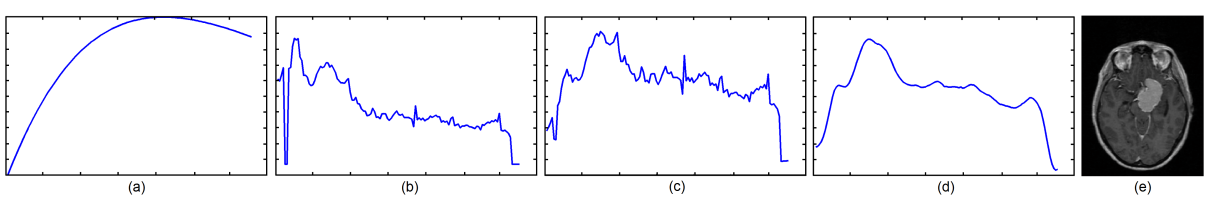

Fig. 4. The complete process of Slice of Interest $E[S]$ extraction: (a) Inverse-Gamma prior $P(Z)$, (b) spatially-constrained EMD asymmetry distance for $S_{Z}$, (c) posterior probability $P\left(Z \mid S_{Z}\right)$, (d) $P\left(Z \mid S_{Z}\right)$ is filtered by Gaussian low-pass filter with $\sigma=3$, (e) the most asymmetrical slice $E[S]$ extracted as the maxima of $P\left(Z \mid S_{Z}\right)$.
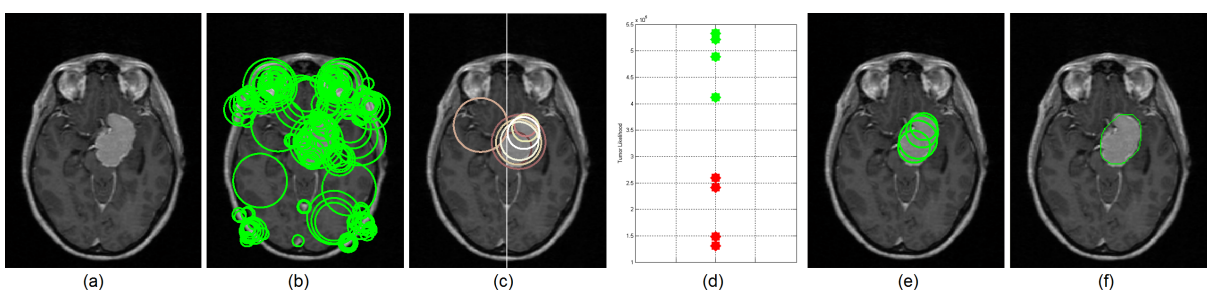

Fig. 5. Case \#1 intermediate results for tumor blob detection: (a) $E[S]$, (b) extracted CSDD blob features $\vec{B}$, (c) the top $5 \%$ most asymmetrical blobs $\vec{B}^{*}$ the whiter the outline represents the higher asymmetrical score, with the extracted MSP as the white vertical line, (d) result of kmeans clustering with y-axis as the tumor-likelihood $P\left(B_{j}^{*}\right)$ for each blob $B_{j}^{*}$, (e) retaining the cluster that yields the higher mean $P\left(B_{j}^{*}\right)$ gives us the true positive tumor blobs $E\left[\overrightarrow{B^{*}}\right]$, (f) the final $2 \mathrm{D}$ rough tumor shape.

noises (Figure 4). Finally we can take the maxima of this signal to be our $E[S]$ and proceed to segment tumor's 2D shape (Figure 4). We define the convulotion of $\mathrm{f}$ and $\mathrm{g}$ as $\mathrm{f} \star \mathrm{g}$, and by setting the parameters $Z=1: z, \mu=0$, and $\sigma=3$, we are able to find the tumor slice:

$$
E[S]=\max \left\{P\left(Z \mid S_{Z}\right) \star N(\mu, \sigma)\right\}
$$

\subsection{Stage 3: Blob Feature Extraction with Asymmetry Processing}

From the extracted slice of interest, we proceed to extract the tumor's $2 \mathrm{D}$ shape with a state of the art blob detector. We use Center-Surround Distribution Distance (CSDD) [4] as our blob and interest region detector, which is insensitive to geometric deformation. CSDD is based on comparing the cumulative distributions of intensity and texture of an extracted region and its surrounding circular background.

$E[S]$ is first smoothed with Gaussian low-pass filter, where $N\left(l=5, \mu=0, \sigma^{2}=\right.$ 2 ) to get rid of possible noise, then CSDD blob features [4] are extracted from the filtered $E[S]$ (Figure 5). We denote the extracted blob features as $\vec{B}$ with each single blob feature denoted as $B_{i}$. To eliminate all the false positives blobs that do not surround the actual tumor, we compute the EMD [17] of the intensity distribution 1D histogram (only intensity information) from the areas enclosed by each blob $B_{i}$ and its corresponding 
MSP-reflection area $\operatorname{Re} f\left(B_{i}\right)$, and retain the highest 5\% EMD metric blobs, denoted as $\overrightarrow{B^{*}}$, in which blobs with higher EMD score imply the enclosed structure as being more asymmetrical.

$$
\overrightarrow{B^{*}}=\max \left\{\Phi\left(H_{1}\left(B_{i}\right), H_{1}\left(\operatorname{Re} f\left(B_{i}\right)\right)\right),\left\lfloor\frac{5 N}{100}\right\rfloor\right\}
$$

We define and compute the tumor-likelihood score $P\left(B_{j}^{*}\right)$ of each blobs $B_{j}^{*}$ of $\overrightarrow{B^{*}}$ by weighting EMD asymmetry score of $B_{j}^{*}$ with its blob strength $S\left(B_{j}^{*}\right)$, which is the EMD measure of how distinctive is the foreground and background intensity of blob $B_{j}^{*}$. Then, we dividing the weighted asymmetry score by its foreground intensity variance $\operatorname{Var}\left[I\left(B_{j}^{*}\right)\right]$, because the non-tumor false positives such as part of the scalp and surrounding tissues may yield high intensity variance, whereas the tumor tissue in an area remains uniform intensity.

$$
P\left(B_{j}^{*}\right)=\frac{S\left(B_{j}^{*}\right) \Phi\left(H_{1}\left(B_{j}^{*}\right), H_{1}\left(\operatorname{Ref}\left(B_{j}^{*}\right)\right)\right)}{\operatorname{Var}\left[I\left(B_{j}^{*}\right)\right]}
$$

K-means clustering [2] algorithm with $k=2$ is then used to cluster $P\left(\overrightarrow{B^{*}}\right)$ into two groups $\vec{B}_{k=1}^{*}$ and $\vec{B}_{k=2}^{*}$, and the expected tumor blobs $E\left[\overrightarrow{B^{*}}\right]$ can be retained by keeping the cluster that yields the highest likelihood (Figure 5).

$$
E\left[\overrightarrow{B^{*}}\right]=\max \left\{\operatorname{avg}\left({\overrightarrow{B^{*}}}_{k=1}\right), \operatorname{avg}\left({\overrightarrow{B^{*}}}_{k=2}\right)\right\}
$$

It is possible that $E\left[\overrightarrow{B^{*}}\right]$ can have blobs at spur and false positive locations instead of one connected component. In which case, we use a heuristic approach to remove outlier blobs by keeping the connected component that has the most blobs with the highest EMD asymmetry score $\Phi\left(H_{1}\left(B_{j}^{*}\right), H_{1}\left(\operatorname{Re} f\left(B_{j}^{*}\right)\right)\right.$. To obtain the final rough 2D location and shape of the tumor from $E\left[\overrightarrow{B^{*}}\right]$, simply segment the combined contour of the blobs $E\left[\overrightarrow{B^{*}}\right]$, which yields the rough $2 \mathrm{D}$ shape of the tumor (Figure 5).

\subsection{Stage 4: 3D Tumor Delineation by IFT (image forest transform) Watershed}

The previous steps provide the approximate location of the tumor and a rough shape of this tumor within the SOI. The next step is the precise delineation of the tumor and this is performed for the whole $3 \mathrm{D}$ image.

The approach we use in this work utilizes markers-controlled watershed, placing object and background seeds within the SOI and letting the watershed grow the regions in 3D. However, there are many different algorithms of watershed and their segmentation results are not the same [1]. In this work, we use the IFT-watershed [18] which is based on the Image Foresting Transform [18]. IFT is a general tool for designing of image processing operators based on connectivity, reducing image processing problems into an optimum path forest problem in a graph derived from the image. We chose the IFTwatershed method because it is considerably faster [18] and implements the watershed 


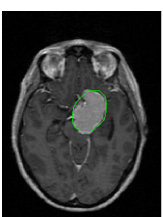

(a)

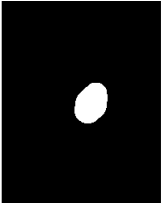

(b)

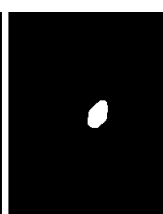

(c)

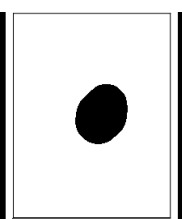

(d)

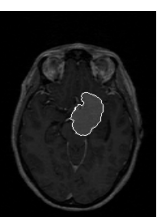

(e)

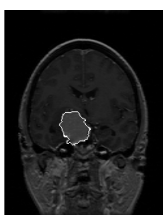

(f)

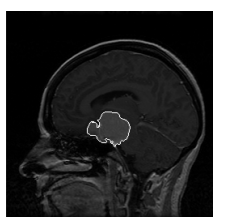

(g)

Fig. 6. IFT-Watershed segmentation process and result. (a) input image, (b) initial mask, (c) tumor seeds, (d) background seeds, (e)-(f) final segmentation in axial, coronal and sagittal orientation, respectively.

in such a way that resolves the "tie zones" dividing them in a balanced manner between the seeds. Further information about the details and evaluation of the IFT-Watershed can be found in [1] and [18].

However, the watershed requires an initialization by placing some object and background seeds, so we developed a way to automatically place these seeds using the result from the blobs extraction stage. In our case, the term "object" below refers to the tumor, and the background refers to everything else.

The result from the blob extraction is a $2 \mathrm{D}$ binary mask (where we have zero for background and one for object). To generate the object seeds, we apply the erosion morphological operator on this mask using a circular structuring element with radius adaptive to the input mask. This morphological operation is performed in $2 \mathrm{D}$ within the SOI. The result is shown in Table 6 . The background seeds are generated in a similar way by applying the dilation operator instead of erosion but then computing the complement of the image (inverting values 0 for 1 and vice-versa), resulting in the image shown in Table 6.

In essence, we create a region of uncertainty around the borders of the mask within the SOI. By definition, the internal seed voxels are already considered to be tumor voxels, and the background seeds are non-tumor voxels. The unmarked voxels are the region of uncertainty which will be resolved by the IFT-Watershed.

Although the seed generation is performed only in one slice (SOI), we let the watershed grow to the rest of the 3D image. Figure 6 shows the resulting segmentation after the IFT-Watershed.

\section{Results}

We test our algorithm on 17 MRI data. The 17 3D MR volumes are T1 weighted and post gadolinium enhanced images acquired in the Axial plane. Other modalities such as T2 or FLAIR are not required by our proposed method.

By visual inspection, the MSP alignments (stage 1) are sucessful for all 17 brain scans in the dataset,which is critical to the success of our symmetry based algorithm. Stage 2 (SOI extraction) located correct slices for 14 out of 17 cases, where stage 3 (2D localization) located the correct tumor location in 13 of 14. Stage 4 (IFT-Watershed) was able to segment the 3D shape of the tumor in all 13 with 1 of which that was not as 

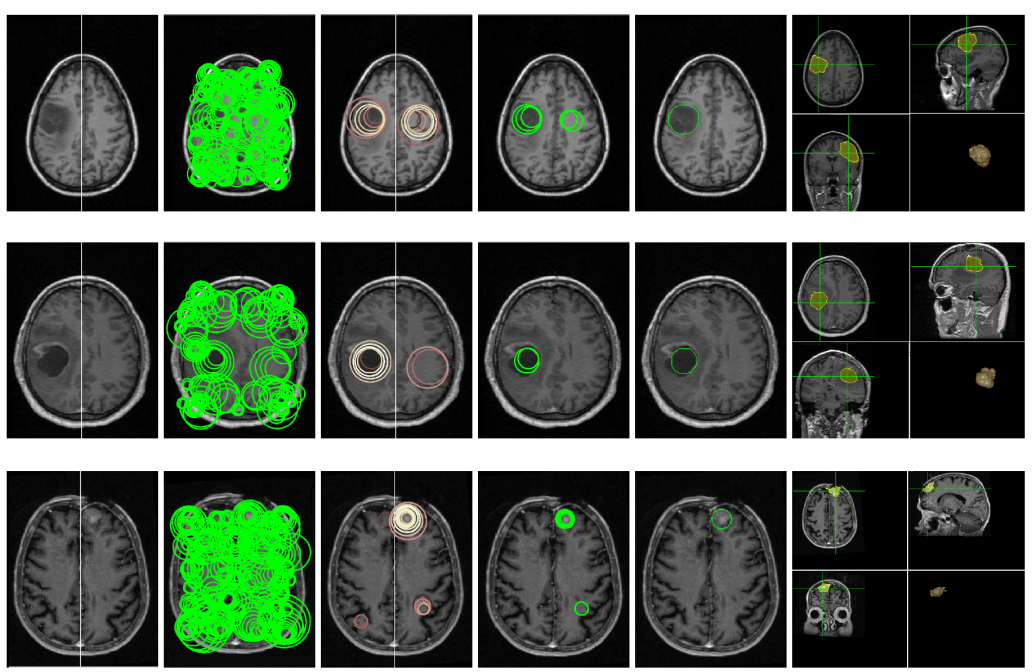

Fig. 7. Sample Results. Row 1 to 3: Case 5, 6, and 11.

good due to complicated tumor tissue. Overall, our stage 3 and stage 4 generates very robust results based on the slice that was extracted from stage 2 .

Quantitative results are calculated using Jaccard Coefficient, where the True Positives (TP) are identified as the overlap between the manually segmented ground truth tumor labels and the machine generated tumor labels.

Jaccard Coefficient:

$$
J C=\frac{T P}{F P+F N+T P}
$$

Our proposed algorithm achieves the mean Jaccard Coefficient of $71.23 \% \pm 27.68 \%$ and the median of $81.68 \%$ from the cases that produced outputs. If we disregard case 17 where it failed miserably and should be considered as a failure case, our mean Jaccard Coefficient is in fact $77.11 \% \pm 18.57 \%$. We also visually inspect the output of each intermediate stages and label the result as either Match and Mismatch to indicate whether or not the tumor has been located. To further demonstrate the robustness of the blob localization (stage 3 ) asymmetry processing, we manually select a tumor slice if stage 2 fails to locate a tumor slice (case 10,12, and 15) and shows very high accuracy. Table 2 shows the complete quantitative results where bold letters indicate manual selected tumor slice in the case of failed stage 2 .

Comparing to other unsupervised and symmetry-based methods [16][22], our proposed method is able to work fully automatically without any user intervention, and is processed fully in 3D. We also achieve a much higher accuracy than what's reported from [22] (highest segmentation score being 71.15\%), with very fast mean run time of under 3 minutes per 3D MR scan, which is very fast comparing to recent publications in both supervised and unsupervised related work. 


\begin{tabular}{|c|c|c|c|c|c|c|c|c|c|}
\hline \multicolumn{10}{|c|}{ Post MSP Extraction 3-Stage Result for 17 Cases } \\
\hline Case \# & Stg 1 & $\operatorname{Stg} 2$ & time $(\mathrm{s})$ & Stg 3 & time (s) & Stg 4 & time (s) & Total run-time $(\mathrm{s})$ & Jcrd Coeff $(\%)$ \\
\hline 1 & 1.47 & In & 108.44 & Auto & 48.48 & Success & 40.28 & 197.2 & 93.99 \\
\hline 2 & 0.85 & In & 104.35 & Auto & 76.34 & Success & 43.17 & 223.86 & 88.07 \\
\hline 3 & 2.47 & In & 114.24 & Auto & 81.03 & Success & 42.61 & 237.88 & 73.92 \\
\hline 4 & 2.76 & In & 111.36 & Auto & 79.23 & Success & 38.01 & 228.6 & 72.61 \\
\hline 5 & 2.10 & In & 98.02 & Auto & 65.12 & Success & 42.35 & 205.49 & 81.68 \\
\hline 6 & 3.08 & In & 113.25 & Auto & 79.21 & Success & 38.55 & 231.01 & 90.11 \\
\hline 7 & 1.97 & In & 94.55 & Auto & 69.49 & Success & 35.29 & 199.33 & 31.74 \\
\hline 8 & 1.14 & In & 106.41 & Auto & 73.88 & Success & 44.78 & 225.07 & 54.10 \\
\hline 9 & 1.15 & In & 51.57 & Auto & 42.51 & Success & 26.42 & 120.50 & 92.61 \\
\hline 10 & 1.39 & Out & 61.74 & Manual & 51.66 & Success & 34.21 & 147.61 & 87.47 \\
\hline 11 & 1.02 & In & 42.66 & Auto & 47.60 & Success & 19.34 & 109.6 & 69.85 \\
\hline 12 & 1.29 & Out & 44.93 & Manual & 62.97 & Success & 29.05 & 136.95 & 90.57 \\
\hline 13 & 0.37 & In & 55.89 & Auto & 57.90 & Success & 26.93 & 140.72 & 92.66 \\
\hline 14 & 1.23 & In & 104.53 & Auto & 88.29 & Success & 36.18 & 229.00 & 83.98 \\
\hline 15 & 0.97 & Out & 30.35 & Manual & 62.81 & Success & 13.59 & 106.75 & 90.73 \\
\hline 16 & 1.49 & In & 33.03 & Auto & 57.23 & Fail & 20.31 & 110.57 & 0.00 \\
\hline 17 & 0.47 & In & 68.6 & Auto & 74.67 & Fail & 24.89 & 168.16 & 0.61 \\
\hline Mean & \multicolumn{3}{|c|}{$79.06 \pm 3$} & $65.79=$ & -13.45 & \multicolumn{2}{|l|}{32.70} & $177.55 \pm 49.47$ & $71.23 \pm 27.68$ \\
\hline
\end{tabular}

Table 1. Results for the 4-Stage on all 17 Cases

\section{Conclusion}

We have proposed a novel unsupervised statistical asymmetry-based, automatic tumor segmentation framework. The method combines a set of state of the art computer vision techniques and has been validated on 17 clinical MR images. Our future work will focus on developing robust segmentation methods for more challenging cases where small, multiple tumors, and diffused boundaries are present.

\section{Acknowledgement}

This work is supported in part by a Grace Woodward grant for collaborative research in engineering and medicine at PSU and by CNPq agency. We also gratefully acknowledge that some of the brain scans used in this paper are provided by Dr. R. Kikinis's lab.

\section{References}

1. Audigier, R., Lotufo, R. A.: Watershed by Image Foresting Transform, Tie-Zone, and Theoretical Relationships with Other Watershed Definitions. In: Proceedings of the 8th International Symposium on Mathematical Morphology (2007)

2. Alsabti, K., Ranka, S., Singh, V.: An efficient k-means clustering algorithm. In: Proc. 1st Workshop on High Performance Data Mining (1998)

3. Cobzas, D., Birkbeck, N., Schmidt, M., Jagersand, M., Murtha, A.: 3D Variational Brain Tumor Segmentation using a High Dimensional Feature Set. ICCV (2007)

4. Collins, R., Ge, W.: CSDD Features: Center-Surround Distribution Distance for Feature Extraction and Matching. ECCV (2008)

5. Corso, J.J., Sharon, E., Yuille, A.: Multilevel Segmentation and Integrated Bayesian Model Classification with an Application to Brain Tumor Segmentation. MICCAI (2006) 
6. Bergo, F.P., Falcao, A., Yasuda, C., Ruppert, G.: FAST AND ROBUST MID-SAGITTAL PLANE LOCATION IN 3D MR IMAGES OF THE BRAIN: Biomedical Engineering Systems and Technologies, vol. 25, pp. 278-290 (2008)

7. Gering, D.T.: Diagonalized nearest neighbor pattern matching for brain tumor segmentation. MICCAI (2003)

8. Iftekharuddin, K.M., Zheng, J., Islam, M.A., Ogg, R.J.: Fractal-based brain tumor detection in multimodal MRI. AMC (2008)

9. Joshi, S., Lorenzen, P., Gerig, G., Bullitt, E.:Structural and radiometric asymmetry in brain images. Medical Image Analysis, 7(2): 155-170 (2003)

10. Klein, A., Andersson, J., Ardekani B.A., Ashburner J., Avants B., Chiang M.C., Christensen G.E., Collins D.L., Gee J., Hellier P., Song J.H., Jenkinson M., Lepage C., Rueckert D., Thompson P., Vercauteren T., Woods R.P., Mann J.J., Parsey R.V.: Evaluation of 14 nonlinear deformation algorithm applied to human brain MRI registration. Neuroimage (2009)

11. Kumar, S., Hebert, M.: Discriminative fields for modeling spatial dependencies in natural images. NIPS (2003)

12. Lafferty, J., Pereira, F., McCallum, A.: Conditional random fields: Probabilistic models for segmenting and labeling sequence data. ICML (2001)

13. Lee, C.H., Greiner, R., Schmidt, M.: Support vector random fields for spatial classification. In: PKDD, pp. 121-132 (2005)

14. Lee, C.H., Wang, S., Murtha, A., Brown, M.R.G., Greiner, R.: Segmenting Brain Tumors using Pseudo-Conditional Random Fields. MICCAI (2008)

15. Li, S.Z.: Markov Random Field Modeling in Image Analysis. Springer-Verlag, Tokyo (2001)

16. Mancas, M., Gosselin, B., and Macq,B.: Fast and automatic tumoral area localization using symmetry. IEEE International Conference on Acoustics, Speech and Signal Processing, 2: 725-728 (2005)

17. Ling, H., Okada, K.: An Efficient Earth Mover's Distance Algorithm for Robust Histogram Comparison. PAMI (2007)

18. Lotufo, R., Falcao, A.: The ordered queue and the optimality of the watershed approaches, In: Mathematical Morphology and its Applications to Image and Signal Processing, vol. 18, pp. 341-350 (2000)

19. Ray, N., Saha, B., and Brown, M.: Locating Brain Tumors from MR Imagery Using Symmetry. ACSSC (2007).

20. Najnam, L., Couprie, M.: Watershed algorithms and contrast preservation. In: Lecture notes in computer science, vol 2886, pp. 62V71 (2003)

21. Prastawa, M., Bullitt, E., Gerig, G.: A Brain Tumor Segmentation Framework Based on Outlier Detection. Medical Image Analysis, vol 150 (2004)

22. Ray, N., Saha, B., Brown, M.:Locating Brain Tumors from MR Imagery Using Symmetry. ACSSC (2007)

23. Schmidt, M., Levner, I., Greiner, R., Murtha, A., Bistritz, A.: Segmenting brain tumors using alignment-based features. MLA (2005)

24. Ruppert, G. C. S., Teverovskiy, L., Yu, C., Falcao, A. X., Liu, Y.: A New Symmetry-based Method for Mid-sagittal Plane Extraction in Neuroimages. International Symposium on Biomedical Imaging: From Macro to Nano (2011)

25. Volkau, I., Prakash, K. N. B., Ananthasubramaniam, A., Aziz, A. and Nowinski, W. L.: Extraction of the midsagittal plane from morphological neuroimages using the KullbackLeibler's measure. In: Medical Image Analysis, 10(6): 863-874, (2006)

26. Zhang, J., Ma, K., Er, M., Chong, V.: Tumor Segmentation from Magnetic Resonance Imaging by Learning via One-Class Support Vector Machine. IWAIT (2004)

27. Koshy, D., Yu, C., Nguyen, D., Kashyap, S., Collins, R., Liu, Y.: Supervised Machine Learning for Brain Tumor Detection in Structural MRI. In: Radiological Society of North America, RSNA (2011) 